\title{
Linear and nonlinear ultraparabolic equations of Kolmogorov type arising in diffusion theory and in finance
}

\author{
Ermanno Lanconelli, Andrea Pascucci and Sergio Polidoro \\ Dipartimento di Matematica, Università di Bologna *†
}

Devoted to Olga A. Ladyzhenskaya in occasion of her 80th birthday

\begin{abstract}
This paper contains a survey on a series of papers by the authors, dealing with linear and non linear Kolmogorov-type operators, arising in diffusion theory, probability and finance. Some new results, about existence for Cauchy problems, regularity properties and pointwise estimates of solutions, are also announced.
\end{abstract}

\section{The Kolmogorov equation and the rank condition}

In its simplest form, Kolmogorov's equation can be written as

$$
\partial_{x_{1}}^{2} u+x_{1} \partial_{x_{2}} u-\partial_{t} u=0, \quad\left(x_{1}, x_{2}, t\right)=(x, t) \in \mathbb{R}^{2} \times \mathbb{R} .
$$

The second order part in (1.1) is strongly degenerate due to the presence in it of the only term $\partial_{x_{1}}^{2}$. However Kolmogorov constructed already in 1934 an explicit fundamental solution of (1.1) which is a $C^{\infty}$ function outside the diagonal [24]. This implies that (1.1) is hypoelliptic, i.e. every distributional solution to (1.1) in an open subset $\Omega$ of $\mathbb{R}^{3}$ actually is a $C^{\infty}(\Omega)$ function.

As Hörmander pointed out in the introduction of his celebrated paper on hypoelliptic second order differential equations [22], the Komogorov method can also be applied to the more general operator

$$
L=\operatorname{div}(A D)+\langle x, B D\rangle-\partial_{t}, \quad x \in \mathbb{R}^{N}, t \in \mathbb{R},
$$

where $D=\left(\partial_{x_{1}}, \ldots, \partial_{x_{N}}\right)$ and $\langle\cdot, \cdot\rangle$ denote, respectively, the gradient and the inner product in $\mathbb{R}^{N} ; A=\left(a_{i j}\right)$ and $B=\left(b_{i j}\right)$ are $N \times N$ constant real matrices, and $A$ is symmetric and non-negative defined. We remark that equation (1.1) can be written in the form (1.2) with

$$
A=\left(\begin{array}{ll}
1 & 0 \\
0 & 0
\end{array}\right) \quad \text { and } \quad B=\left(\begin{array}{ll}
0 & 1 \\
0 & 0
\end{array}\right)
$$

*Piazza di Porta S. Donato 5, 40126 Bologna (Italy). E-mail: lanconel@dm.unibo.it, pascucci@dm.unibo.it, polidoro@dm.unibo.it

${ }^{\dagger}$ Investigation supported by the University of Bologna. Funds for selected research topics. 
For any operator of the type (1.2), we denote, for every $t \in \mathbb{R}$,

$$
E(t)=\exp \left(-t B^{T}\right)
$$

and

$$
C(t)=\int_{0}^{t} E(s) A E^{T}(s) d s
$$

It is not difficult to show that

$$
C(t)>0 \text { for every } t>0 \text {, }
$$

if and only if the following condition holds

$\operatorname{Ker}(A)$ does not contain non-trivial subspaces which are invariant for $B$.

Under condition (1.6), following Kolmogorov's method, Hörmander constructed in [22], page 148, (see also [25], Theorems 1 and 4) an explicit fundamental solution for (1.2):

$$
\Gamma(x, t, \xi, \tau)=\Gamma(x-E(t-\tau) \xi, t-\tau),
$$

where $\Gamma(x, t)=0$ if $t \leq 0$ and

$$
\Gamma(x, t)=\frac{(4 \pi)^{-\frac{N}{2}}}{\sqrt{\operatorname{det} C(t)}} \exp \left(-\frac{1}{4}\left\langle C^{-1}(t) x, x\right\rangle-t \operatorname{tr}(B)\right), \quad \text { if } t>0 .
$$

Hereafter we use the notations

$$
z=(x, t), \zeta=(\xi, \tau), \quad x, \xi \in \mathbb{R}^{N}, t, \tau \in \mathbb{R} .
$$

It is quite trivial to recognize that $\Gamma(z, \zeta)$ is a $C^{\infty}$ function outside $\left\{(z, \zeta) \in \mathbb{R}^{N+1} \times \mathbb{R}^{N+1}\right.$ : $z=\zeta$ \}. Then, under condition (1.6), the operator $L$ in (1.2) is hypoelliptic. It is noteworthy to remark that condition (1.6) can also be expressed in geometric-differential form. Indeed, if we set

$$
X_{j}=\sum_{k=1}^{N} a_{j k} \partial_{x_{k}}, j=1, \ldots, N, \quad Y=\langle x, B D\rangle-\partial_{t},
$$

then (1.6) is equivalent to the following condition

$$
\operatorname{rank} \operatorname{Lie}\left(X_{1}, \ldots, X_{N}, Y\right)=N+1 \text {, }
$$

at any point of $\mathbb{R}^{N+1}$. In (1.10), Lie $\left(X_{1}, \ldots, X_{N}, Y\right)$ denotes the Lie algebra generated by $X_{1}, \ldots, X_{N}, Y$. For an easy proof of the equivalence of $(1.5),(1.6)$ and $(1.10)$, see Theorem 3 in [25] and Proposition A.1 in [31].

In PDE's theory, rank conditions like (1.10) are today called of Hörmander's type because of the following celebrated result. Consider a second order differential operator

$$
P u:=\sum_{j=1}^{p} X_{j}^{2} u+X_{0} u+c u
$$


where

$$
X_{j}=\sum_{k=1}^{N} a_{j k} \partial_{x_{k}}, j=1, \ldots, p,
$$

and the $a_{j k}$ are real valued $C^{\infty}$ functions in an open set $\Omega \subseteq \mathbb{R}^{n}$. Hörmander discovered that $P$ is hypoelliptic in $\Omega$ if

$$
\operatorname{rank} \operatorname{Lie}\left(X_{1}, \ldots, X_{p}, X_{0}\right)=n,
$$

at any point of $\Omega([22]$, Theorem 1.1$)$.

Hörmander's Theorem was extended by Radkevič [45] and by Oleǐnik and Radkevič [35] to general second order operators with smooth coefficients and non-negative characteristic form

$$
L=\sum_{i, j=1}^{N} \partial_{x_{i}}\left(a_{i j} \partial_{x_{j}}\right)+\sum_{i=1}^{N} b_{i} \partial_{x_{i}}+c .
$$

If the coefficients $a_{i j}, b_{i}, c$ are real analytic, then the rank condition is also necessary for the hypoellipticity of $L$ (see [15] and [35], Chap. II, Sec. 8).

\section{Lie group and metric structures related to Kolmogorov op- erators}

Let $L$ be the operator in (1.2) with constant matrices $A$ and $B$. In [31] it was shown that $L$ is invariant with respect to the left translations of the Lie group $\mathcal{G}=\left(\mathbb{R}^{N+1}, \circ\right)$ with composition law defined by

$$
(x, t) \circ(\xi, \tau)=(\xi+E(\tau) x, t+\tau), \quad(x, t),(\xi, \tau) \in \mathbb{R}^{N} \times \mathbb{R},
$$

with $E(\cdot)$ as in (1.3). It is easily checked that in $\mathcal{G}$

$$
(\xi, \tau)^{-1}=(-E(-\tau) \xi,-\tau)
$$

then, by (1.7), we can write

$$
\Gamma(z, \zeta)=\Gamma\left(\zeta^{-1} \circ z\right)
$$

The Hörmander condition (1.10) implies that, for some basis on $\mathbb{R}^{N}$, the matrices $A$ and $B$ take the following block form

$$
A=\left(\begin{array}{cc}
A_{0} & 0 \\
0 & 0
\end{array}\right)
$$

and

$$
\left(\begin{array}{ccccc}
* & B_{1} & 0 & \cdots & 0 \\
* & * & B_{2} & \cdots & 0 \\
\vdots & \vdots & \vdots & \ddots & \vdots \\
* & * & * & \cdots & B_{r} \\
* & * & * & \cdots & *
\end{array}\right)
$$


where $A_{0}$ is a symmetric non-singular $p_{0} \times p_{0}$ matrix and the $B_{j}$ 's blocks are $p_{j-1} \times p_{j}$ matrices of rank $p_{j}, j=1,2, \ldots, r$. The $p_{j}$ 's are positive integers such that

$$
p_{0} \geq p_{1} \geq \ldots p_{r} \geq 1, \quad \text { and } \quad p_{0}+p_{1}+\cdots+p_{r}=N
$$

and the blocks denoted by $*$ are arbitrary (see [31], Proposition 2.1).

In the sequel we shall call Kolmogorov operator with constant coefficients any operator of the type (1.2) with the matrices $A$ and $B$ satisfying the above structural conditions. The class of Kolmogorov operators with constant coefficients contains a remarkable subclass of operators which are also invariant with respect to a suitable dilation group. Indeed, there exists a group of dilations $\left(\delta_{\lambda}\right)_{\lambda>0}$ such that

$$
L \circ \delta_{\lambda}=\lambda^{2}\left(\delta_{\lambda} \circ L\right), \quad \forall \lambda>0,
$$

if and only if all the $*$-blocks in (2.3) are zero matrices. In this case

$$
\delta_{\lambda}=\operatorname{diag}\left(\lambda I_{p_{0}}, \lambda^{3} I_{p_{1}}, \ldots, \lambda^{2 r+1} I_{p_{r}}, \lambda^{2}\right),
$$

where $I_{p_{j}}$ denotes the $p_{j} \times p_{j}$ identity matrix. The proofs of these statements are contained in [27] and [31] (cf. Proposition 2.2).

When the $*$-blocks in $B$ are zero, the dilations $\left(\delta_{\lambda}\right)_{\lambda>0}$ in (2.5) are a group of automorphisms of $\mathcal{G}$. Equipped with them, $\mathcal{G}$ becomes a homogeneous group with homogeneous dimension $Q+2$, where

$$
Q:=p_{0}+3 p_{1}+\cdots+(2 r+1) p_{r},
$$

(see [26], page 288, and [31], Remark 2.1).

We shall call homogeneous Kolmogorov operator every Kolmogorov operator whose matrix $B$ has null $*$-blocks.

It is esay to check that the fundamental solution $\Gamma$ of a homogeneous Kolmogorov equation is $\delta_{\lambda}$-homogeneous of degree $-Q$, i.e.

$$
\Gamma\left(\delta_{\lambda}(z)\right)=\lambda^{-Q} \Gamma(z), \quad \forall z \in \mathbb{R}^{N+1} \backslash\{0\}, \forall \lambda>0 .
$$

In this case $\Gamma$ takes the following simple form:

$$
\Gamma(x, t)=\frac{c_{N}}{t^{Q / 2}} \exp \left(-\frac{1}{4}\left\langle C^{-1}(1) D_{0}\left(t^{-1 / 2}\right) x, D_{0}\left(t^{-1 / 2}\right) x\right\rangle\right),
$$

where $C(1)$ is given by (1.4), with $t=1$ and $D_{0}(\lambda)$ is the $N \times N$ matrix

$$
D_{0}(\lambda)=\operatorname{diag}\left(\lambda I_{p_{0}}, \lambda^{3} I_{p_{1}}, \ldots, \lambda^{2 r+1} I_{p_{r}}\right) .
$$

Moreover $c_{N}=(4 \pi)^{-N / 2}(\operatorname{det} C(1))^{-1 / 2}$ (see [31], Proposition 2.3).

In the family of Kolmogorov operators, the homogeneous ones play a central role. Indeed, any Kolmogorov operator can be approximated, in a suitable sense, by a homogeneous operator. More precisely, let $B$ in (2.3) be the matrix related to some Kolmogorov operator with constant coefficients

$$
L=\operatorname{div}(A D)+\langle x, B D\rangle-\partial_{t} .
$$


If we denote by $B_{0}$ the matrix obtained by annihilating every $*$-block in (2.3), the operator

$$
L_{0}=\operatorname{div}(A D)+\left\langle x, B_{0} D\right\rangle-\partial_{t}
$$

is homogeneous. Denoting by $\Gamma$ and $\Gamma_{0}$ the fundamental solutions with pole at $\zeta=0$ of $L$ and $L_{0}$ respectively, then, for every $b>0$ there exists a positive constant $a$ such that

$$
\frac{1}{a} \Gamma_{0}(z) \leq \Gamma(z) \leq a \Gamma_{0}(z)
$$

on the level set $\left\{z: \Gamma_{0}(z)>b\right\}$ (see [31], Theorem 3.1). The operator $L_{0}$ is homogeneous and, due to inequalities (2.7), it could be called the principal part of $L$.

As we already noticed, the fundamental solution of a constant coefficients Kolmogorov operator $L$ is invariant with respect to the left translations on $\mathcal{G}=\left(\mathbb{R}^{N+1}, \circ\right)$, and homogeneous of degree $-Q$ with respect to the dilations $\delta_{\lambda}\left(\mathcal{G}\right.$ and $\delta_{\lambda}$ defined in (2.1) and (2.5)). Then, it is quite obvious to expect that the intrinsic geometry underlying $L$ is that one determined by $\mathcal{G}$ and $\delta_{\lambda}$.

Let $\alpha_{1}, \ldots, \alpha_{N}$ be strictly positive integers such that

$$
\delta_{\lambda}=\operatorname{diag}\left(\lambda^{\alpha_{1}}, \ldots, \lambda^{\alpha_{N}}, \lambda^{2}\right)
$$

and define, for every $z \in \mathbb{R}^{N+1} \backslash\{0\},\|z\|_{\mathcal{G}}=\varrho$ where $\varrho$ is the unique positive solution to the equation

$$
\frac{t^{2}}{\varrho^{4}}+\sum_{j=1}^{N} \frac{x_{j}^{2}}{\varrho^{2 \alpha_{j}}}=1, \quad z=\left(x_{1}, \ldots, x_{N}, t\right) .
$$

We agree to let $\|z\|_{\mathcal{G}}=0$ if $z=0$. Then

$$
z \longmapsto\|z\|_{\mathcal{G}}
$$

is a $\delta_{\lambda}$-homogeneous function of degree one, continuous on $\mathbb{R}^{N+1}$, strictly positive and of class $C^{\infty}$ in $\mathbb{R}^{N+1} \backslash\{0\}$. If we define

$$
d_{\mathcal{G}}(z, \zeta)=\left\|\zeta^{-1} \circ z\right\|_{\mathcal{G}}, \quad z, \zeta \in \mathbb{R}^{N+1}
$$

then $\left(\mathbb{R}^{N+1}, d_{\mathcal{G}}\right)$ is a (pseudo-)metric space, the natural one for the operator $L$. Indeed, it is not difficult to recognize that $d_{\mathcal{G}}$ is equivalent to the control distance related to $L$, which is defined as follows. Let $Z_{1}, \ldots, Z_{N}$ be the column vectors of the square root of $A$. We agree to identify $Z_{j}$ with the vector $\left(Z_{j}, 0\right)$ of $\mathbb{R}^{N+1}$. Let us also identify the first order differential operator $Y$ with the vector field $\left(B^{T} x,-1\right)$. We call $L$-admissible curve every absolutely continuous function

$$
\gamma:[0,1] \longrightarrow \mathbb{R}^{N+1}
$$

such that

$$
\dot{\gamma}(s)=\sum_{j=1}^{N} c_{j}(s) Z_{j}+c_{N+1}(s) Y(\gamma(s)) \quad \text { a.e. in }[0,1] \text {. }
$$


Let us denote

$$
I(\gamma)=\int_{0}^{1}\left(\sum_{j=1}^{N}\left(c_{j}(s)\right)^{4}+\left(c_{N+1}(s)\right)^{2}\right)^{\frac{1}{4}} d s .
$$

The rank condition (1.6) is equivalent to the following one

$$
\operatorname{rank} \operatorname{Lie}\left(Z_{1}, \ldots, Z_{N}, Y\right)=N+1
$$

at any point of $\mathbb{R}^{N+1}$. Thanks to a classical theorem of Caratheodory-Razewski-Chow, this condition implies that every point $\zeta \in \mathbb{R}^{N+1}$ is reachable from an arbitrary given point $z \in \mathbb{R}^{N+1}$ by means of $L$-admissible curves. Then

$$
d_{L}(z, \zeta)=\inf \{I(\gamma) \mid \gamma L \text {-admissible, } \gamma(0)=z, \gamma(1)=\zeta\}
$$

is finite for every $z, \zeta \in \mathbb{R}^{N+1}$. The function

$$
(z, \zeta) \longmapsto d_{L}(z, \zeta)
$$

actually is a distance in $\mathbb{R}^{N+1}$, called $L$-control distance. Since $Z_{1}, \ldots, Z_{N}$ and $Y$ are invariant with respect to the left translations on $\mathcal{G}$ and $\delta_{\lambda}$-homogeneous of degree one and two respectively, we have

$$
d_{L}(z, \zeta)=d_{L}\left(\zeta^{-1} \circ z, 0\right)=: d_{L}\left(\zeta^{-1} \circ z\right)
$$

and

$$
d_{L}\left(\delta_{\lambda}(z)\right)=\lambda d_{L}(z)
$$

These properties easily imply that

$$
\frac{1}{a} d_{L}(z, \zeta) \leq d_{\mathcal{G}}(z, \zeta) \leq a d_{L}(z, \zeta), \quad \forall z, \zeta \in \mathbb{R}^{N+1},
$$

for a suitable constant $a>0$.

We want to stress that the composition law (2.1), the dilations (2.5) and the metric $d_{\mathcal{G}}$ are determined only by the matrix $B$, i.e. by the first order part of the operator $L$. We would like to mention an observation contained in the recent paper [7].

Remark 2.1 If the $*$-blocks in $B$ are zero and we define

$$
\widetilde{\delta}_{\lambda}:=\operatorname{diag}\left(\lambda I_{p_{0}}, \lambda^{2} I_{p_{1}}, \ldots, \lambda^{r+1} I_{p_{r}}, \lambda\right), \quad \lambda>0,
$$

then $\left(\widetilde{\delta}_{\lambda}\right)_{\lambda>0}$ is another group of automorphisms of $\mathcal{G}$. Equipped with these dilations, $\mathcal{G}$ becomes a homogeneous Carnot group and

$$
\Delta_{\mathcal{G}}=\operatorname{div}(A D)+Y^{2}
$$

is its intrinsic sub-Laplacian (here $Y$ is the first order differential operator defined in (1.9)). 


\section{Some motivation for studying Kolmogorov equations}

The operator (1.1) in previous section is the lowest dimension version of the following degenerate parabolic operator in $\mathbb{R}^{N+1}, N=2 n$,

$$
L=\sum_{j=1}^{n} \partial_{x_{j}}^{2}+\sum_{j=1}^{n} x_{j} \partial_{x_{n+j}}-\partial_{t}
$$

Kolmogorov introduced (3.1) in 1934 in order to describe the probability density of a system with $2 n$ degree of freedom. The $2 n$-dimensional space is the phase space, $\left(x_{1}, \ldots, x_{n}\right)$ is the velocity and $\left(x_{n+1}, \ldots, x_{2 n}\right)$ the position of the system. By choosing

$$
A=\left(\begin{array}{cc}
I_{n} & 0 \\
0 & 0
\end{array}\right) \quad \text { and } \quad B=\left(\begin{array}{cc}
0 & I_{n} \\
0 & 0
\end{array}\right)
$$

where $I_{n}$ and 0 denote respectively the identity and the null $n \times n$ matrices, operator (3.1) can be written as in (1.2). We also recall that (3.1) is a prototype for a family of evolution equations arising in the kinetic theory of gases that take the following general form

$$
Y u=\mathcal{J}(u) .
$$

Here $\mathbb{R}^{2 n} \ni x \longmapsto u(x, t) \in \mathbb{R}$ is the density of particles which have velocity $\left(x_{1}, \ldots, x_{n}\right)$ and position $\left(x_{n+1}, \ldots, x_{2 n}\right)$ at time $t$,

$$
Y u:=-\sum_{j=1}^{n} x_{j} \partial_{x_{n+j}} u+\partial_{t} u
$$

is the so called total derivative of $u$ and $\mathcal{J}(u)$ describes some kind of collisions. This last term can take different form, either linear or non linear. For instance, in the usual Fokker-Planck equation, we have

$$
\mathcal{J}(u)=\sum_{i, j=1}^{n} a_{i j} \partial_{x_{i} x_{j}}^{2} u+\sum_{i=1}^{n} a_{i} \partial_{x_{i}} u+a u
$$

where $a_{i j}, a_{i}$ and $a$ are functions of $(x, t) ; \mathcal{J}(u)$ may also occur in divergence form

$$
\mathcal{J}(u)=\sum_{i, j=1}^{n} \partial_{x_{i}}\left(a_{i j} \partial_{x_{j}} u+b_{i} u\right)+\sum_{i=1}^{n} a_{i} \partial_{x_{i}} u+a u .
$$

We also mention the following non linear collision operator of Fokker-Planck-Landau type

$$
\mathcal{J}(u)=\sum_{i, j=1}^{n} \partial_{x_{i}}\left(a_{i j}(z, u) \partial_{x_{j}} u+b_{i}(z, u)\right)
$$

where the coefficients $a_{i j}$ and $b_{i}$ depend on $z \in \mathbb{R}^{2 n+1}$ and on the unknown function $u$ through some integral expressions. This operator is studied as a simplified version of the Boltzmann 
collision operator. For the description of wide classes of stochastic processes and kinetic models leading to equations of the previous type, we refer to the classical monographies [9], [16] and [10].

Ultraparabolic differential equations with non linear total derivative terms appear when studying convection-diffusion models. We would like to mention the paper by Escobedo, Vázquez and Zuazua [18] in which the following equation is studied

$$
\partial_{y} g(u)-\partial_{t} u=-\Delta_{x} u, \quad x=\left(x_{1}, \ldots, x_{n}\right) \in \mathbb{R}^{n}, y, t \in \mathbb{R} .
$$

The linearized equation of (3.6)

$$
g^{\prime}(u) \partial_{y} v-\partial_{t} v=-\Delta_{x} v
$$

if $g^{\prime}(u)$ is different from zero and smooth enough, can be reduced to the form (1.2) with $N=n+2$,

$$
A=\left(\begin{array}{cccc}
1 & \cdots & 0 & 0 \\
\vdots & \ddots & \vdots & \vdots \\
0 & \cdots & 1 & 0 \\
0 & \cdots & 0 & 0
\end{array}\right) \quad \text { and } \quad B=\left(\begin{array}{cccc}
0 & \cdots & 0 & 1 \\
\vdots & \ddots & \vdots & \vdots \\
0 & \cdots & 0 & 0 \\
0 & \cdots & 0 & 0
\end{array}\right)
$$

We would also like to mention the paper [36] where blow-up phenomena of Fujita type are studied for semilinear equations related to Kolmogorov operators.

In the last decades mathematical models involving linear and non linear Kolmogorov type equations have also appeared in finance [1], [4], [5] and [49]. We explicitly mention the equation

$$
s^{2} \partial_{s}^{2} V+(\log s) \partial_{\tau} V+\partial_{t} V=0, \quad s>0, \tau, t \in \mathbb{R}
$$

which arises in the problem of pricing Asian options. (3.8) can be reduced to the Kolmogorov equation (1.1) by means of an elementary change of variables (see [6], page 479). Very recently, the nonlinear Kolmogorov type equation

$$
\partial_{x_{1}}^{2} u+u \partial_{x_{2}} u-\partial_{t} u=f
$$

has been proposed in [2] as a mathematical model for utility functional and decision making. When $f=0,(3.9)$ becomes a particular case of (3.6).

\section{Linear equations with Hölder continuous coefficients}

For linear Kolmogorov operators with Hölder continuous coefficients very satisfactory results are present in literature. Weber [48] in 1951, Il'in [23] in 1964 and Sonin [47] in 1967 constructed a fundamental solution for linear Kolmogorov operators. Regularity results of solutions and first boundary value problems were investigated by Genčev [21], Šatyro [46], Eidelman, Ivasyshen and Malytska [17]. The results of these authors have been generalized and improved in a series of papers by Polidoro [40, 41, 42], Manfredini [33] and Lunardi [32]. 
Consider in $\mathbb{R}^{N+1}$ the second order differential operator

$$
L=\sum_{i, j=1}^{p_{0}} a_{i j}(z) \partial_{x_{i} x_{j}} u+\langle x, B D u\rangle-\partial_{t} u,
$$

where $1 \leq p_{0} \leq N, z=(x, t) \in \mathbb{R}^{N} \times \mathbb{R}$. Assume the matrix $B$ as in (2.3) with the *blocks equal to zero; the block $B_{j}$ has rank $p_{j}$ and dimension $p_{j-1} \times p_{j}, j=1, \ldots, r$, with $p_{0} \geq p_{1} \geq \cdots \geq p_{r} \geq 1$. Suppose the matrix $\left(a_{i j}\right)_{i, j=1, \ldots, p_{0}}$ in (4.1) uniformly positive definite, i.e. there exists $\lambda>0$ such that

$$
\frac{1}{\lambda} \sum_{j=1}^{p_{0}} \xi_{j}^{2} \leq \sum_{i, j=1}^{p_{0}} a_{i j}(z) \xi_{i} \xi_{j} \leq \lambda \sum_{j=1}^{p_{0}} \xi_{j}^{2}
$$

for every $\left(\xi_{1}, \ldots, \xi_{p_{0}}\right) \in \mathbb{R}^{p_{0}}$ and for every $z \in \mathbb{R}^{N+1}$. We also assume the $a_{i j}$ Hölder continuous with exponent $\alpha \in] 0,1\left[\right.$ with respect to the distance $d_{\mathcal{G}}$ in (2.8), i.e.

$$
\left|a_{i j}(z)-a_{i j}(\zeta)\right| \leq M d_{\mathcal{G}}(z, \zeta)^{\alpha}, \quad \forall z, \zeta \in \mathbb{R}^{N+1},
$$

for some constant $M$. Under these hypotheses, in [40] the following theorem is proved

Theorem 4.1 The operator $L$ in (4.1) has a global fundamental solution $\Gamma$ satisfying the following lower and upper local estimates: for every $\varepsilon>0$ there exists $k>0$ such that

$$
(1-\varepsilon) Z(z, \zeta) \leq \Gamma(z, \zeta) \leq(1+\varepsilon) Z(z, \zeta),
$$

for any $z, \zeta \in \mathbb{R}^{N+1}$ such that $Z(z, \zeta) \geq k$. In (4.4), $Z(z, \zeta)$ denotes the parametrix of $L$ with pole at $\zeta$, i.e. the fundamental solution with pole at $\zeta$ of the frozen operator

$$
L_{\zeta}=\sum_{i, j=1}^{p_{0}} a_{i j}(\zeta) \partial_{x_{i} x_{j}}+\langle x, B D\rangle-\partial_{t} .
$$

We remark that $Z(z, \zeta)$ takes the explicit Gaussian type form (1.7)-(1.8), where $C(t)$ is given by (1.4) with $A=\left(\alpha_{i j}\right)_{i, j=1, \ldots, N}, \alpha_{i j}=a_{i j}(\zeta)$ if $1 \leq i, j \leq p_{0}$ and $\alpha_{i j}=0$ otherwise.

Theorem 4.1, which in particular improves and generalizes the previous results by Weber [48], Il'in [23] and Sonin [47], was proved in [40] by adapting the Levi's parametrix method to the Lie group and metric structures related to the matrix $B$ (cf. Section 2).

The Levi's parametrix method also provides a global upper bound for the fundamental solution $\Gamma$. It was shown in [40], Corollary 2.5, that there exists a positive constant $\lambda$ such that, if $\Gamma^{+}$denotes the fundamental solution of the constant coefficients Kolmogorov operator

$$
L^{+}=\lambda \Delta_{p_{0}}+\langle x, B D\rangle-\partial_{t}
$$

then, for every $T>0$, there exists a positive constant $c^{+}=c^{+}(T)$ with the property that

$$
\Gamma(z, \zeta) \leq c^{+} \Gamma^{+}(z, \zeta)
$$


for every $z=(x, t), \zeta=(\xi, \tau) \in \mathbb{R}^{N+1}, 0<t-\tau<T$.

If the operator $L$ can be written in divergence form, a lower bound for $\Gamma$ analogous to (4.6) also holds. This result relies on the local estimates (4.4) of $\Gamma$ and on a Harnack inequality for non negative solutions to $L u=0$, which is invariant with respect to the translation and dilation groups, related to the matrix $B$, described in Section 2. To be more specific, let us introduce some notation. Consider the Euclidean cylinder

$$
H_{1}=\left\{(x, t) \in \mathbb{R}^{N} \times \mathbb{R}|| x|<1,| t \mid<1\right\} .
$$

For every $z_{0}=\left(x_{0}, t_{0}\right) \in \mathbb{R}^{N+1}$ and $r>0$, we set

$$
H_{r}\left(z_{0}\right) \equiv z_{0} \circ\left(\delta_{r}\left(R_{1}\right)\right)=\left\{z \in \mathbb{R}^{N+1} \mid z=z_{0} \circ \delta_{r}(\zeta), \zeta \in R_{1}\right\}
$$

and

$$
H_{r}^{-}\left(z_{0}\right)=\left\{z \in H_{r}\left(z_{0}\right) \mid t=t_{0}-r^{2}\right\} .
$$

Then, the following theorem holds (see [40]).

Theorem 4.2 Let $L$ as in Theorem 4.1 and assume it can be written in divergence form:

$$
L=\operatorname{div}(A D)+\langle x, B D\rangle-\partial_{t} .
$$

Let $\Omega$ be an open subset of $\mathbb{R}^{N+1}$. Then there exist constants $c_{0}, r_{0}>0$ and $\left.\theta \in\right] 0,1[$ only depending on $B$ and on the constants $\lambda$ and $M$ in (4.2)-(4.3) such that

$$
\sup _{H_{r \theta}^{-}\left(z_{0}\right)} u \leq c_{0} u\left(z_{0}\right)
$$

for every non negative solution $u$ to $L u=0$ in $\Omega$ and for every $H_{r}\left(z_{0}\right) \subseteq \Omega, 0<r \leq r_{0}$.

We would like to precise the meaning of solution to $L u=0$. A continuous function

$$
u: \Omega \longrightarrow \mathbb{R}, \quad \Omega \subseteq \mathbb{R}^{N+1},
$$

is a solution to $L u=0$ in $\Omega$ if each derivative $\partial_{x_{i}} u, \partial_{x_{i} x_{j}}^{2} u$, for $1 \leq i, j \leq p_{0}$, and

$$
Y u:=\langle x, B D u\rangle-\partial_{t} u
$$

exists in weak sense and it is a continuous function. Moreover

$$
\sum_{i, j=1}^{p_{0}} a_{i j}(z) \partial_{x_{i} x_{j}} u(z)-Y u(z)=0, \quad \forall z \in \Omega .
$$

Remark 4.3 By using the embedding Theorem of Folland [19], one can see that every solution to Lu $=0$ actually is Hölder continuous with respect to the norm $\|\cdot\|_{\mathcal{G}}$.

Theorem 4.2 extends some Harnack inequalities for constant coefficients Kolmogorov operators first appeared in [28], [20] and [31].

Starting from Theorem 4.2, the following global lower bound estimate is proved (see [42], Main Theorem). 
Theorem 4.4 Let $L$ as in Theorem 4.2. Then there exists a positive constant $\lambda$ such that, if $\Gamma^{-}$denotes the fundamental solution of

$$
L^{-}=\lambda^{-1} \Delta_{p_{0}}+\langle x, B D\rangle-\partial_{t},
$$

then, for every $T>0$, there exists a positive constant $c^{-}$with the property that

$$
c^{-} \Gamma^{-}(z, \zeta) \leq \Gamma(z, \zeta)
$$

for every $z=(x, t), \zeta=(\xi, \tau) \in \mathbb{R}^{N+1}, 0<t-\tau<T$.

We would like again to stress that the functions $\Gamma^{-}$and $\Gamma^{+}$appearing in (4.6) and (4.8) have the explicit form (1.7)-(1.8), with the matrix $A$ in (1.4) replaced by $\lambda^{-1} \operatorname{diag}\left(I_{p_{0}}, 0, \ldots, 0\right)$ and $\lambda \operatorname{diag}\left(I_{p_{0}}, 0, \ldots, 0\right)$ respectively.

Theorem 4.4 was proved in [42] by using a rather complicated technique which was inspired by a method of Aronson and Serrin for classical parabolic operators. The core of the method used in [42] is a kind of discretization of the connectivity Theorem of Caratheodory-RazewskiChow. The rank condition

$$
\operatorname{rank} \operatorname{Lie}\left(\partial_{x_{1}}, \ldots, \partial_{x_{p_{0}}}, Y\right)=N+1, \quad \text { at any point of } \mathbb{R}^{N+1}
$$

played a crucial role.

The estimates (4.6) and (4.8) were then used to prove uniqueness and representation theorems for solutions to the Cauchy problem related to $L$ (see Theorem 3.1 in [41]).

Theorem 4.5 Let $L$ as in Theorem 4.2. Let $u \in C\left(\mathbb{R}^{N} \times[0, T], \mathbb{R}\right)$ be a solution of the Cauchy problem

$$
\left\{\begin{array}{l}
\left.L u=0 \quad \text { in } \mathbb{R}^{N} \times\right] 0, T[ \\
u(\cdot, 0)=0 .
\end{array}\right.
$$

Then $u \equiv 0$ if one of the following condition is satisfied.

(i) There exists $c>0$ such that

$$
\int_{\left.\mathbb{R}^{N} \times\right] 0, T[} e^{-c|x|^{2}} u(x, t) d x d t<\infty ;
$$

(ii) $u \geq 0$.

Positive solutions to Kolmogorov equations are of special interest since they have the role of distribution functions in several stochastic models. In these contexts, the following representation and Fatou type results contained in the same paper cited above, [41] Theorem 4.1, are of some relevance.

Theorem 4.6 Let $L$ as in Theorem 4.2. Then:

(i) for every non negative solution to $L u=0$ in $\left.\mathbb{R}^{N} \times\right] 0, T[$ there exists a nonnegative Borel measure $\mu$ such that

$$
\int_{\mathbb{R}^{N}} e^{-c|x|^{2}} d \mu(x)<\infty
$$


for some positive constant $c$, and

$$
\left.u(z)=\int_{\mathbb{R}^{N}} \Gamma(z,(\xi, 0)) d \mu(\xi), \quad \forall z \in \mathbb{R}^{N} \times\right] 0, T[
$$

(ii) for every non negative Borel measure $\mu$ verifying (4.9) there exists $T>0$ such that the function $u$ in (4.10) is a solution to Lu=0 in $\left.\mathbb{R}^{N} \times\right] 0, T[$. Moreover

$$
\lim _{t \rightarrow 0^{+}} u(\cdot, t)=\mu \quad \text { in measure }
$$

and

$$
\lim _{t \rightarrow 0^{+}} u(x, t)=\varphi(x) \quad \text { a.e. }
$$

where $\varphi d x$ is the absolutely continuous part of $\mu$ with respect to the Lebesgue measure.

We want to close this section by briefly recalling the interior Schauder type estimates proved in [33] (see also [32]). The results of these papers improve and generalizes the previous ones contained in [21], [46] and [17].

Let $L$ as in Theorem 4.2 and $u$ a smooth real function defined on a subset $\Omega$ of $\mathbb{R}^{N+1}$. Then, for every bounded open set $\Omega_{1}$ such that $\bar{\Omega}_{1} \subseteq \Omega$, there exists a constant $c>0$ such that

$$
|u|_{2+\alpha, \Omega_{1}} \leq c\left(\sup _{\Omega}|u|+|L u|_{\alpha, \Omega}\right) .
$$

Here we use the notations

$$
|f|_{\alpha, \Omega}=\sup _{\substack{z \neq \zeta \\ z, \zeta \in \Omega}} \frac{|f(z)-f(\zeta)|}{\left(d_{\mathcal{G}}(z, \zeta)\right)^{\alpha}}
$$

and

$$
|f|_{2+\alpha, \Omega_{1}}=\sum_{i, j=1}^{p_{0}}\left|\partial_{x_{i} x_{j}} f\right|_{\alpha, \Omega_{1}}+|Y u|_{\alpha, \Omega_{1}}+\sup _{\Omega_{1}}|u| .
$$

In [33], the interior Schauder estimates are also used to study a first boundary value problem for $L$.

We would also like to quote the paper [29] in which a boundary value problem for a class of quasilinear operators of Fokker-Planck type was studied. In [29] the a priori estimates of [33] are used as crucial tools.

\section{Linear equations with non-continuous coefficients}

A priori $L^{p}$ estimates and Hölder regularity properties of solutions to Kolmogorov equations with weakly continuous coefficients have been proved in [8], [34], [43] and [44]. Let $L$ be the following operator

$$
L=\sum_{i, j=1}^{p_{0}} a_{i j}(z) \partial_{x_{i} x_{j}}+\langle x, B D\rangle-\partial_{t}
$$


Suppose the matrix $\left(a_{i j}\right)_{i, j=1, \ldots, p_{0}}$ satisfies the positivity condition (4.2) and that the constant matrix $B$ is as in (2.3) with all the $*$-blocks equal to zero and the $B_{j}$ 's of maximum rank. We also assume the coefficients $a_{i j}$ satisfying the following weak continuity condition

$$
a_{i j} \in \mathrm{VMO}_{\mathcal{G}}, \quad i, j=1, \ldots, p_{0} .
$$

The space of vanishing mean oscillation $\mathrm{VMO}_{\mathcal{G}}$ is defined as follows. Denote by $B_{r}\left(z_{0}\right)$ the $d_{\mathcal{G}}$-ball of center $z_{0}$ and radius $r$ :

$$
B_{r}\left(z_{0}\right)=\left\{z \in \mathbb{R}^{N+1} \mid d_{\mathcal{G}}\left(z_{0}, z\right)<r\right\} .
$$

Here $d_{\mathcal{G}}$ stands for the distance defined in (2.8), Section2. We say that $u \in \mathrm{VMO}_{\mathcal{G}}$ if: $u \in L_{\mathrm{loc}}^{1}\left(\mathbb{R}^{N+1}\right)$ and

$$
\lim _{r \rightarrow 0^{+}}\left(\sup _{\varrho \leq r} \frac{1}{\operatorname{meas}\left(B_{\varrho}\right)} \int_{B_{\varrho}}\left|u-u_{B_{\varrho}}\right|\right)=0
$$

where

$$
u_{B_{\varrho}}=\frac{1}{\operatorname{meas}\left(B_{\varrho}\right)} \int_{B_{\varrho}} u(\zeta) d \zeta
$$

Here meas $\left(B_{\varrho}\right)$ denotes the Lebesgue measure of $B_{\varrho}$ : we want to stress that

$$
\operatorname{meas}\left(B_{\varrho}\right)=\varrho^{Q+2} \operatorname{meas}\left(B_{1}\right) .
$$

In [8] and [43], interior regularity properties of strong solutions to the equation $L u=f$ in $\Omega \subseteq \mathbb{R}^{N+1}$, with $L$ as in (5.1), were studied. In order to recall those results, we need to introduce some notation. If $\Omega$ is an open subset of $\mathbb{R}^{N+1}, 1<q<\infty$ and $0 \leq \mu \leq Q+2$, we set

$$
L^{q, \mu}(\mathcal{G}, \Omega)=\left\{u \in L_{\mathrm{loc}}^{q}(\Omega) \mid\|u\|_{L^{q, \mu}(\mathcal{G}, \Omega)}<\infty\right\}
$$

where

$$
\|u\|_{L^{q, \mu}(\mathcal{G}, \Omega)}^{q}=\sup _{r>0, z \in \Omega} \frac{1}{r^{\mu}} \int_{\Omega \cap B_{r}(z)}|u(\zeta)|^{q} d \zeta .
$$

Obviously, $L^{q, \mu}=L^{q}$ if $\mu=0$. The Sobolev-Morrey type space $S^{q, \mu}(\mathcal{G}, \Omega)$ is defined as follows

$$
S^{q, \mu}(\mathcal{G}, \Omega)=\left\{u \in L^{q, \mu}(\mathcal{G}, \Omega) \mid \partial_{x_{i}} u, \partial_{x_{i} x_{j}} u, Y u \in L^{q, \mu}(\mathcal{G}, \Omega)\right\},
$$

where, as usual, $Y=\langle x, B D\rangle-\partial_{t}$. The norm in this space is defined by

$$
\|u\|_{S^{q, \mu}(\mathcal{G}, \Omega)}^{q}:=\|u\|_{q, \mu}^{q}+\sum_{i=1}^{p_{0}}\left\|\partial_{x_{i}} u\right\|_{q, \mu}^{q}+\sum_{i, j=1}^{p_{0}}\left\|\partial_{x_{i} x_{j}} u\right\|_{q, \mu}^{q}+\|Y u\|_{q, \mu}^{q},
$$

where $\|\cdot\|_{q, \mu}=\|\cdot\|_{L^{q, \mu}(\mathcal{G}, \Omega)}$. When $L$ is as in (5.1), we call strong solution to

$$
L u=f, \quad f \in L^{q, \mu}(\mathcal{G}, \Omega),
$$

a function $u \in S^{q, \mu}(\mathcal{G}, \Omega)$ satisfying (5.5) pointwise a.e. in $\Omega$. Then, the following theorem holds (see [43], Theorems 1.5 and 1.6): 
Theorem 5.1 Let $u$ be a strong solution to (5.5) in $\Omega$ with $L$ in the non-divergence form (5.1). Then, for every open set $\Omega_{1} \subset \subset \Omega$, there exists a positive constant $c$ which does not depend on $u$, such that

$$
\|u\|_{S^{q, \mu}(\mathcal{G}, \Omega)} \leq c\left(\|f\|_{L^{q, \mu}(\mathcal{G}, \Omega)}+\|u\|_{L^{q, \mu}(\mathcal{G}, \Omega)}\right) .
$$

Moreover, if $2 q+\mu>Q+2$ then $u$ is $\mathcal{G}$-Hölder continuous with exponent $\alpha=\min \left\{1, q^{-1}(2 q+\right.$ $\mu-Q-2)\}$, i.e.

$$
|u(z)-u(\zeta)| \leq c\left(d_{\mathcal{G}}(z, \zeta)\right)^{\alpha}\left(\|f\|_{L^{q, \mu}(\mathcal{G}, \Omega)}+\|u\|_{L^{q, \mu}(\mathcal{G}, \Omega)}\right),
$$

for every $z, \zeta \in \Omega_{1}$. Finally, if $\beta=q^{-1}(q+\mu-Q-2)>0$, then

$$
\left|\partial_{x_{i}} u(z)-\partial_{x_{i}} u(\zeta)\right| \leq c\left(d_{\mathcal{G}}(z, \zeta)\right)^{\beta}\left(\|f\|_{L^{q, \mu}(\mathcal{G}, \Omega)}+\|u\|_{L^{q, \mu}(\mathcal{G}, \Omega)}\right),
$$

for every $z, \zeta \in \Omega_{1}, i=1, \ldots, p_{0}$.

We stress that the constant $c$ in the provious inequalities depend on the " $\mathrm{VMO}_{\mathcal{G}}$ continuity moduli" of the coefficients $a_{i j}$.

Inequality (5.6) in the case $\mu=0$ was first proved in [8]. The technique used in [8] and [43] is inspired to the one first introduced by Chiarenza, Frasca and Longo [11] for classical elliptic operators in non divergence form. The starting point is a representation formula which, in the present context, takes the following form. Let $u \in C_{0}^{\infty}\left(\mathbb{R}^{N+1}\right)$ and $i, j=1, \ldots, p_{0}$. Then

$$
\begin{aligned}
\partial_{x_{i} x_{j}} u(z)= & -\mathrm{P} . \mathrm{V} . \int_{\mathbb{R}^{N+1}} \Gamma_{i j}\left(z ; \zeta^{-1} \circ z\right) \sum_{h, k=1}^{p_{0}}\left(a_{h k}(z)-a_{h k}(\zeta)\right) \partial_{x_{h} x_{k}} u(\zeta) d \zeta \\
& -L u(z) \int_{\|\zeta\|_{\mathcal{G}}=1} \Gamma_{j}(z ; \zeta) \nu_{j}(\zeta) d \sigma(\zeta) .
\end{aligned}
$$

Here $\Gamma(z ; \cdot)$ denotes the fundamental solution with pole at $z$ of the constant coefficients Kolmogorov operator

$$
L_{z}=\sum_{i, j=1}^{p_{0}} a_{i j}(z) \partial_{\xi_{i}} \xi_{j}-\langle\xi, B D\rangle-\partial_{t} .
$$

Moreover $\Gamma_{j}=\partial_{\xi_{j}} \Gamma, \Gamma_{i j}=\partial_{\xi_{i} \xi_{j}} \Gamma$ and $\nu=\left(\nu_{1}, \ldots, \nu_{N+1}\right)$ is the outer normal to $\left\{\|\zeta\|_{\mathcal{G}}=1\right\}$. Finally

$$
\text { P. V. } \int_{\mathbb{R}^{N+1}} f=\lim _{\varepsilon \rightarrow 0} \int_{\|\zeta\|_{\mathcal{G}} \geq \varepsilon} f
$$

The key tools used in [8] and [43] are some deep continuity results for singular integrals, and their commutators with $\mathrm{VMO}_{\mathcal{G}}$ functions, modelled on the Lie group and metric structures described in Section 2.

The same methods and techniques, suitably adapted, were used in [34] and in [44] in order to prove interior regularity results for weak solutions to the divergence form equation

$$
L u:=\sum_{i, j=1}^{p_{0}} \partial_{x_{i}}\left(a_{i j}(z) \partial_{x_{j}}\right)+Y u=\sum_{i=1}^{p_{0}} \partial_{x_{i}} F_{i}(z)
$$


with $a_{i j} \in \mathrm{VMO}_{\mathcal{G}}$ and $F_{i} \in L_{\text {loc }}^{q}\left(\mathbb{R}^{N+1}\right)$. A weak solution to (5.7) in an open set $\Omega \subseteq \mathbb{R}^{N+1}$ is a function $u \in L_{\text {loc }}^{2}(\Omega)$ such that the weak derivatives $\partial_{x_{1}} u, \ldots, \partial_{x_{p_{0}}} u, Y u$ exist and belong to $L_{\mathrm{loc}}^{2}(\Omega)$ and

$$
\int_{\Omega}\left(\sum_{i, j=1}^{p_{0}} a_{i j} \partial_{x_{i}} u \partial_{x_{j}} \psi-(Y u) \psi\right) d z=\int_{\Omega} \sum_{i=1}^{p_{0}} F_{i} \partial_{x_{i}} \psi d z,
$$

for every $\psi \in C_{0}^{\infty}(\Omega)$. The main results in [34] and [44] are summarized in the following theorem.

Theorem 5.2 Let $u$ be a weak solution to (5.7). Assume the matrix $\left(a_{i j}\right)$ satisfies the positivity condition (4.2) and the weak continuity property (5.2). We also assume the matrix Bsatisfies the above structural hypotheses. Then, for every open set $\Omega_{1} \subset \subset \Omega, 1<q<\infty$, $0 \leq \mu<Q+2$, there exists a positive constant $c=c\left(\Omega_{1}, \Omega, L, q\right)$ such that

$$
\left\|\partial_{x_{j}} u\right\|_{L^{q, \mu}\left(\mathcal{G}, \Omega_{1}\right)} \leq c\left(\|u\|_{L^{q, \mu}(\mathcal{G}, \Omega)}+\sum_{i=1}^{p_{0}}\left\|F_{i}\right\|_{L^{q}(\mathcal{G}, \Omega)}\right),
$$

for every $j=1, \ldots, p_{0}$. Moreover, if $p>Q+2-\mu$, then

$$
|u(z)-u(\zeta)| \leq c\left(d_{\mathcal{G}}(z, \zeta)\right)^{\alpha}\left(\|u\|_{L^{q, \mu}(\mathcal{G}, \Omega)}+\sum_{i=1}^{p_{0}}\left\|F_{i}\right\|_{L^{q}(\mathcal{G}, \Omega)}\right),
$$

for every $z, \zeta \in \Omega_{1}$. Here

$$
\alpha=1-\frac{Q+2-\mu}{q} .
$$

We want to stress again that the constant $c$ in (5.8) depends on the " $\mathrm{VMO}_{\mathcal{G}}$ moduli" of the coefficients $a_{i j}$.

The above Hölder estimates for weak solutions to (5.7) have been used in [30] for studying a boundary value problem for the non linear equation

$$
\sum_{i, j=1}^{p_{0}} \partial_{x_{i}}\left(a_{i j}(z, u) \partial_{x_{j}}\right)+Y u=0 .
$$

However, the dependence of the constant $c$ in (5.8) on the regularity of the coefficients $a_{i j}$ forces quite restrictive hypotheses on the nonlinearity. In order to remove such restrictions, regularity results for solutions to linear equations with merely measurable $a_{i j}$ 's are needed. For instance, when studying existence problems for non linear Kolmogorov-Fokker-Planck equations as in (3.3) and (3.5), pointwise properties of the weak solutions to (5.9) with $a_{i j} \in L_{\text {loc }}^{\infty}$ could be crucial. A first result in such a direction have been very recently proved by the second and the third author. In [38], they prove the local boundedness of the weak solutions to (5.9) only assuming the uniform positivity condition (4.2) for the matrix $\left(a_{i j}\right)$. The main result in [38] is the following theorem. 
Theorem 5.3 Suppose

$$
\frac{1}{\lambda}|\xi|^{2} \leq \sum_{i, j=1}^{p_{0}} a_{i j}(z) \xi_{i} \xi_{j} \leq \lambda|\xi|^{2}
$$

for every $\xi=\left(\xi_{1}, \ldots, \xi_{p_{0}}\right) \in \mathbb{R}^{p_{0}}$ and for every $z \in \mathbb{R}^{N+1}$. Let $u$ be a weak solution to

$$
\sum_{i, j=1}^{p_{0}} \partial_{x_{i}}\left(a_{i j}(z) \partial_{x_{j}}\right)+Y u=0
$$

in an open set $\Omega \subseteq \mathbb{R}^{N+1}$. Then we have

$$
\sup _{H_{\varrho}\left(z_{0}\right)}|u| \leq c\left(\frac{1}{(r-\varrho)^{Q+2}} \int_{\frac{1}{H_{r}\left(z_{0}\right)}}|u|^{p}\right)^{\frac{1}{p}},
$$

for every $p \geq 1$ and $r, \varrho>0$ such that $\frac{r}{2} \leq \varrho<r$ and $\overline{H_{r}\left(z_{0}\right)} \subseteq \Omega$. Here $H_{r}\left(z_{0}\right)$ is the $\mathcal{G}$-cylinder defined in (4.7). The constant $c$ only depends on $p, \lambda$ and the matrix $B$.

This theorem is proved in [38] by using an iterative procedure analogous to the one introduced by Moser in the classical elliptic and parabolic cases. As it is well known, the Moser technique is based on a combination of Caccioppoli type estimates with the classical Sobolev inequality. Now, the weak solutions to (5.10) satisfy a Caccioppoli type estimate. However, it only gives $L_{\text {loc }}^{2}$ bound of the first order derivatives $\partial_{x_{j}} u, j=1, \ldots, p_{0}$ and does not give any information on the others $\left(N-p_{0}\right)$ spatial derivatives. Thus, if $p_{0}<N$, this lack of information cannot be restored by the usual Sobolev embedding theorem.

The key idea in [38] is to prove a Sobolev type inequality for non negative sub-and supersolutions to (5.10), good enough to be successfully combined with the previous "weak" Caccioppoli inequality. To be more specific, let us first recall the definition of weak sub- and super-solution to (5.10). We say that a function $u \in L_{\text {loc }}^{2}(\Omega), \Omega$ open subset of $\mathbb{R}^{N+1}$, is a weak sub-solution to (5.10) if the weak derivatives $\partial_{x_{1}} u, \ldots, \partial_{x_{p_{0}}} u, Y u$ exist, belong to $L_{\text {loc }}^{2}(\Omega)$ and

$$
\int_{\Omega}-\langle A D u, D \varphi\rangle+\varphi Y u \geq 0, \quad \forall \varphi \in C_{0}^{\infty}(\Omega), \varphi \geq 0 .
$$

If $-u$ is a weak sub-solution, we say that $u$ is a weak super-solution. Then, the following Caccioppoli type estimate holds (cf. [38], Proposition 3.2)

Theorem 5.4 Let $u$ be a non-negative weak sub-solution of (5.10) in $\Omega$. Let $\varrho, r>0$, $\frac{r}{2} \leq \varrho<r$, and $\overline{H_{r}} \subseteq \Omega$. Then, there exists a constant $c$, only dependent on $\lambda$ in (4.2) and on the homogeneous dimension $Q$, such that

$$
\left\|\partial_{x_{j}} u^{p}\right\|_{L^{2}\left(H_{\varrho}\right)} \leq \frac{c \sqrt{1+\varepsilon}}{\varepsilon}\left\|u^{p}\right\|_{L^{2}\left(H_{r}\right)}, \quad \text { where } \varepsilon=\frac{|2 p-1|}{4 p},
$$

for every $j=1, \ldots, p_{0}$ and $p<0$ or $p \geq 1$. The same inequality holds for non-negative weak super-solutions and $p \in] 0,1 / 2[$. 
The key Sobolev type inequality for weak sub- and super-solutions proved in [38] is the following.

Theorem 5.5 Let $u$ be a non-negative weak sub-solution to (5.10) and let $r, \varrho$ be as in the previous Theorem 5.4. Then $u \in L_{\mathrm{loc}}^{2 \kappa}\left(H_{\varrho}\right), \kappa=1+\frac{2}{Q}$, and there exists a constant $c$, only dependent on $Q$ and $\lambda$, such that

$$
\|u\|_{L^{2 \kappa}\left(H_{\varrho}\right)} \leq \frac{c}{r-\varrho}\left(\|u\|_{L^{2}\left(H_{r}\right)}+\sum_{j=1}^{p_{0}}\left\|\partial_{x_{j}} u\right\|_{L^{2}\left(B_{r}\right)}\right) .
$$

The same inequality holds for non-negative super-solutions.

Inequalities (5.11)-(5.12) allow to start up an iterative procedure like to the classical Moser's one and to prove Theorem 5.3.

\section{Ultraparabolic equations with non linear total derivatives terms}

We end this survey by recalling some recent results concerning the Kolmogorov equation with a non linear total derivative term

$$
\Delta_{x} u+h(u) \partial_{y} u-\partial_{t} u=f(\cdot, u), \quad(x, y, t) \in \mathbb{R}^{p_{0}} \times \mathbb{R} \times \mathbb{R} .
$$

As said before, this equation arises in combustion theory, see [18], and in mathematical finance, see [2].

Due to the lack of diffusion in the $y$-direction, (6.1) has mixed parabolic and hyperbolic features. Indeed, when $h(u)=u, f \equiv 0$ and the solution only depends on $y$, it becomes the Burger's equation. For this reason, Escobedo, Vazquez and Zuazua [18] consider the Cauchy problem related to (6.1) in the framework of the conservation laws, and prove that, under a suitable entropy condition, there exists a unique distributional solution which is not necessarily a continuous function. On the other hand, Antonelli, Barucci and Mancino in [2] find a (local in time) Hölder continuous viscosity solutions to that Cauchy problem.

The existence and regularity problem for the above weak solutions has been studied in [14], [37], [39]. These results improve and generalize the preceding ones in [18] and [2]. In [14] the following natural definition of classical solution to (6.1) is introduced:

We say that $u$ is a classical solution to the equation in (6.1) if:

(i) $\partial_{x_{i} x_{i}} u, i=1, \ldots, p_{0}$ exists and it is a continuous function;

(ii) the directional derivative

$$
\frac{\partial u}{\partial \nu_{z}}(z), \quad \nu_{z}=(0, h(u(z)),-1)
$$

exists at every point and it is a continuous function of $z=(x, y, t)$;

(iii) equation (6.1) is satisfied at every point. 
The main idea in the study of the regularity of the solutions to (6.1) is a modification of the classical freezing method. To be more specific, if $h$ is a Lipschitz continuous function, we consider

$$
L_{\bar{z}}=\Delta_{x} u+\left(h(u(\bar{z}))+x_{1}-\bar{x}_{1}\right) \partial_{y} u-\partial_{t} u
$$

which is a "good" approximation to the left hand side of (6.1). Note that, up to a straightforward change of coordinates, $L_{\bar{z}}$ is the Kolmogorov operator (1.2) with $N=p_{0}+1$ and the matrices $A, B$ as in (3.7).

It has to be noticed that the rank condition (1.10) is satisfied. Then $L_{\bar{z}}$ has a fundamental solution which takes the explicit form (1.7)-(1.8). Starting with this remark and by using analysis on Lie groups combined with standard techniques in degenerate parabolic problems, in [39] the following existence and uniqueness theorem is proved:

Theorem 6.1 Let $f, g$ and $h$ be Lipschitz continuous in their domains. Then, there exists $T>0$ and a unique function $\left.u: \mathbb{R}^{N} \times\right] 0, T[\longrightarrow \mathbb{R}$, classical solution to

$$
\left\{\begin{array}{l}
\left.\Delta_{x} u+h(u) \partial_{y} u-\partial_{t} u=f(\cdot, u) \quad \text { in } \mathbb{R}^{N} \times\right] 0, T[ \\
u(\cdot, 0)=g
\end{array}\right.
$$

and such that

$$
\begin{aligned}
\left|u(x, y, t)-u\left(x^{\prime}, y^{\prime}, t\right)\right| & \leq c_{0}\left(\left|x-x^{\prime}\right|+\left|y-y^{\prime}\right|\right), \\
\left|u(x, y, t)-u\left(x, y, t^{\prime}\right)\right| & \leq c_{0}\left|t-t^{\prime}\right|^{\frac{1}{2}}(1+|x|+|y|),
\end{aligned}
$$

for every $(x, y, t),\left(x^{\prime}, y^{\prime}, t\right),\left(x, y, t^{\prime}\right) \in \mathbb{R}^{p_{0}} \times \mathbb{R} \times \mathbb{R}$.

A weaker version of Theorem 6.1 was previously proved in [3] by some probabilistic technique.

Further regularity properties of the solution found in [39] can be obtained under some additional condition. We would like only mention the following optimal regularity result which follows from Theorem 3.1 in [37].

Theorem 6.2 Let $u$ be a classical solution to the equation (6.1) in an open set $\Omega \subseteq \mathbb{R}^{p_{0}} \times$ $\mathbb{R} \times \mathbb{R}$. If $f$ and $h$ are $C^{\infty}$ functions on their domains, and $b^{\prime}(u) D_{x} u \neq 0$ at any point of $\Omega$, where $D_{x}=\left(\partial_{x_{1}}, \ldots, \partial_{x_{p_{0}}}\right)$, then

$$
u \in C^{\infty}(\Omega) .
$$

This theorem is an extension of a previous result in [14] and it is proved by using a suitable freezing method introduced by Citti [12] in a different context. Such a method is based on the following remark. Let us define

$$
X_{j}=\partial_{x_{j}}, j=1, \ldots, p_{0}, \text { and } Z=h(u) \partial_{y}-\partial_{t} .
$$

Then, if

$$
b^{\prime}(u) D_{x} u \neq 0, \quad \text { at any point of } \Omega
$$

the condition

$$
\operatorname{dim}\left(\operatorname{span}\left\{X_{1}, \ldots, X_{p_{0}}, Z,\left[X_{1}, Z\right], \ldots,\left[X_{p_{0}}, Z\right]\right\}\right)=p_{0}+2
$$


holds everywhere in $\Omega$. In (6.4), $\left[X_{j}, Z\right]$ denotes the Lie bracket of $X_{j}$ and $Z$. Condition (6.4), which is a kind of Hörmander rank condition of step two, in [37] and [14] is the starting point of a bootstrap argument in suitable spaces of Hölder continuous functions. These spaces are modeled on the vector fields $X_{1}, \ldots, X_{p_{0}}$ and $Z$ in (6.3), and depend on the function $u$. We directly refer to [37] and [14] for more details on such a bootstrap argument which has been used in [12] and [13] to prove the $C^{\infty}$ smoothness of solutions to a Levi curvature equation.

\section{References}

[1] B. Alziary, J. P. DÉcamps, And P. F. Koehl, A P.D.E. approach to Asian options: analytical and numerical evidence, J. Banking Finance, 21 (1997), pp. 613-640.

[2] F. Antonelli, E. Barucci, And M. E. Mancino, Asset pricing with a forwardbackward stochastic differential utility, Econom. Lett., 72 (2001), pp. 151-157.

[3] F. Antonelli And A. Pascucci, On the viscosity solutions of a stochastic differential utility problem, preprint, (2002).

[4] G. BARLES, Convergence of numerical schemes for degenerate parabolic equations arising in finance theory, in Numerical methods in finance, Cambridge Univ. Press, Cambridge, 1997, pp. 1-21.

[5] J. Barraquand and T. Pudet, Pricing of American path-dependent contingent claims, Math. Finance, 6 (1996), pp. 17-51.

[6] E. Barucci, S. Polidoro, And V. Vespri, Some results on partial differential equations and Asian options, Math. Models Methods Appl. Sci., 11 (2001), pp. 475-497.

[7] A. Bonfiglioli and F. Uguzzoni, Families of diffeomorphic sub-Laplacians and free Carnot groups, preprint, (2002).

[8] M. Bramanti, M. C. Cerutti, and M. Manfredini, $L^{p}$ estimates for some ultraparabolic operators with discontinuous coefficients, J. Math. Anal. Appl., 200 (1996), pp. 332-354.

[9] S. Chandresekhar, Stochastic problems in physics and astronomy, Rev. Modern Phys., 15 (1943), pp. 1-89.

[10] S. Chapman and T. G. Cowling, The mathematical theory of nonuniform gases, Cambridge University Press, Cambridge, third ed., 1990.

[11] F. Chiarenza, M. Frasca, And P. Longo, Interior $W^{2, p}$ estimates for nondivergence elliptic equations with discontinuous coefficients, Ricerche Mat., 40 (1991), pp. 149-168.

[12] G. CitTi, $C^{\infty}$ regularity of solutions of a quasilinear equation related to the Levi operator, Ann. Scuola Norm. Sup. Pisa Cl. Sci. (4), 23 (1996), pp. 483-529. 
[13] G. Citti, E. Lanconelli, and A. Montanari, Smoothness of Lipschitz continuous graphs, with non vanishing Levi curvature, Acta Mathematica, to appear (2002).

[14] G. Citti, A. Pascucci, And S. Polidoro, Regularity properties of viscosity solutions of a non-Hörmander degenerate equation, J. Math. Pures Appl. (9), 80 (2001), pp. 901918.

[15] M. DerRidj And C. Zuily, Régularité analytique d'opérateurs dégénérés, C. R. Acad. Sci. Paris Sér. A-B, 273 (1971), pp. A720-A723.

[16] J. J. Duderstadt and W. R. Martin, Transport theory, John Wiley \& Sons, New York-Chichester-Brisbane, 1979. A Wiley-Interscience Publication.

[17] S. D. Eidelman, S. D. Ivasyshen, and H. P. Malytska, A modified Levi method: development and application, Dopov. Nats. Akad. Nauk Ukr. Mat. Prirodozn. Tekh. Nauki, 5 (1998), pp. 14-19.

[18] M. Escobedo, J. L. VÁzquez, And E. Zuazua, Entropy solutions for diffusionconvection equations with partial diffusivity, Trans. Amer. Math. Soc., 343 (1994), pp. 829-842.

[19] G. B. Folland, Subelliptic estimates and function spaces on nilpotent Lie groups, Ark. Mat., 13 (1975), pp. 161-207.

[20] N. Garofalo and E. Lanconelli, Level sets of the fundamental solution and Harnack inequality for degenerate equations of Kolmogorov type, Trans. Amer. Math. Soc., 321 (1990), pp. 775-792.

[21] T. G. Genčev, On ultraparabolic equations, Dokl. Akad. Nauk SSSR, 151 (1963), pp. 265-268.

[22] L. Hörmander, Hypoelliptic second order differential equations, Acta Math., 119 (1967), pp. 147-171.

[23] A. M. IL'In, On a class of ultraparabolic equations, Dokl. Akad. Nauk SSSR, 159 (1964), pp. 1214-1217.

[24] A. Kolmogorov, Zufllige Bewegungen. (Zur Theorie der Brownschen Bewegung.)., Ann. of Math., II. Ser., 35 (1934), pp. 116-117.

[25] L. P. Kupcov, The fundamental solutions of a certain class of elliptic-parabolic second order equations, Differencial'nye Uravnenija, 8 (1972), pp. 1649-1660, 1716.

[26] - The fundamental solutions of a certain class of elliptic-parabolic second order equations, Differencial'nye Uravnenija, 8 (1972), pp. 1649-1660, 1716.

[27] _ , The mean value property and the maximum principle for second order parabolic equations, Dokl. Akad. Nauk SSSR, 242 (1978), pp. 529-532. 
[28] —, On parabolic means, Dokl. Akad. Nauk SSSR, 252 (1980), pp. 296-301.

[29] E. LANCONElli And F. LASCialfari, A boundary value problem for a class of quasilinear operators of Fokker-Planck type, in Proceedings of the Conference "Differential Equations" (Italian) (Ferrara, 1996), vol. 41 suppl., 1996, pp. 65-84 (1997).

[30] E. Lanconelli and D. Morbidelli, On the Poincaré inequality for vector fields, Ark. Mat., 38 (2000), pp. 327-342.

[31] E. Lanconelli and S. Polidoro, On a class of hypoelliptic evolution operators, Rend. Sem. Mat. Univ. Politec. Torino, 52 (1994), pp. 29-63. Partial differential equations, II (Turin, 1993).

[32] A. LunARDI, Schauder estimates for a class of degenerate elliptic and parabolic operators with unbounded coefficients in $\mathbb{R}^{N}$, Ann. Scuola Norm. Sup. Pisa Cl. Sci. (4), 24 (1997), pp. 133-164.

[33] M. Manfredini, The Dirichlet problem for a class of ultraparabolic equations, Adv. Differential Equations, 2 (1997), pp. 831-866.

[34] M. Manfredini And S. Polidoro, Interior regularity for weak solutions of ultraparabolic equations in divergence form with discontinuous coefficients, Boll. Unione Mat. Ital. Sez. B Artic. Ric. Mat. (8), 1 (1998), pp. 651-675.

[35] O. A. Olěnik And E. V. Radkevič, Second order equations with nonnegative characteristic form, Plenum Press, New York, 1973.

[36] A. PAscucci, Fujita type results for a class of degenerate parabolic operators, Adv. Differential Equations, 4 (1999), pp. 755-776.

[37] — Hölder regularity for a Kolmogorov equation, preprint, (2002).

[38] A. Pascucci And S. Polidoro, The Moser's iterative method for a class of ultraparabolic equations, preprint, (2002).

[39] — On the Cauchy problem for a non linear Kolmogorov equation, preprint, (2002).

[40] S. Polidoro, On a class of ultraparabolic operators of Kolmogorov-Fokker-Planck type, Matematiche (Catania), 49 (1994), pp. 53-105 (1995).

[41] — Uniqueness and representation theorems for solutions of Kolmogorov-FokkerPlanck equations, Rend. Mat. Appl. (7), 15 (1995), pp. 535-560 (1996).

[42] — - A global lower bound for the fundamental solution of Kolmogorov-Fokker-Planck equations, Arch. Rational Mech. Anal., 137 (1997), pp. 321-340.

[43] S. Polidoro And M. A. Ragusa, Sobolev-Morrey spaces related to an ultraparabolic equation, Manuscripta Math., 96 (1998), pp. 371-392. 
[44] — Hölder regularity for solutions of ultraparabolic equations in divergence form, Potential Anal., 14 (2001), pp. 341-350.

[45] E. V. Radkevič, Hypoelliptic operators with multiple characteristics, Mat. Sb. (N.S.), 79 (121) (1969), pp. 193-216.

[46] J. I. ŠATYRo, The smoothness of the solutions of certain degenerate second order equations, Mat. Zametki, 10 (1971), pp. 101-111.

[47] I. M. Sonin, A class of degenerate diffusion processes, Teor. Verojatnost. i Primenen, 12 (1967), pp. 540-547.

[48] M. WEBER, The fundamental solution of a degenerate partial differential equation of parabolic type, Trans. Amer. Math. Soc., 71 (1951), pp. 24-37.

[49] P. Wilmott, S. Howison, And J. Dewynne, Option pricing, Oxford Financial Press, Oxford, 1993. 